\title{
Producción científica e impacto del sistema de ciencia de Latinoamérica y el Caribe en revistas de la región
}

\author{
Guillermo Armando Ronda-Pupo*
}

Artículo recibido:

8 de octubre de 2020

Artículo aceptado:

4 de diciembre de 2020

Artículo de investigación

\section{Resumen}

El objetivo del estudio es analizar el ritmo de crecimiento de la producción científica latinoamericana y del Caribe en revistas de la región incluidas en el Journal of Citation Reports de Clarivate Analytics Web of Science y su impacto con la utilización de un modelo alométrico. Se analizaron 179219 documentos publicados entre 2000 y 2017 que recibieron 233352 citas. Para medir el impacto se utilizó una ventana de citaciones fija de tres años $\left(t_{0}+2\right)$. El exponente de la relación resultó $\alpha=0,75 \pm 0.02$ y sugiere que la productividad crece más rápido que el impacto.

\footnotetext{
Universidad de la Frontera, Facultad de Ciencias Jurídicas y Empresariales, Departamento de Administración y Economía, Chile

guillermo.ronda@ufrontera.cl

INVESTIGACIÓN BIBLIOTECOLÓGICA, vol. 35, núm. 88, julio/septiembre, 2021, México, ISSN: 2448-8321 pp. 45-62
} 
Palabras clave: Alometría; Impacto Basado en

Citaciones; Ley de Potencia; Autosemejanza

\title{
Productivity and Citation Impact of the Latin Ameri- can and the Caribbean Science System in domestic journals \\ Guillermo Armando Ronda-Pupo
}

\begin{abstract}
The object of this study is to analyze the growth patterns of the Latin American and Caribean scientific production in the magazines and its citation-based impact using allometric modeling. We analyzed 179219 Latin American documents published from 2000 through 2017 in JCR journals that appear hosted in a Latin American country that received 233352 citations. The citation-based impact was assessed using a three-year fix window of citation $\left(t_{0}+2\right)$. The resulting exponent was $\alpha=0,75 \pm 0.02$, suggesting that scientific production grows faster than its impact.
\end{abstract}

Keywords: Allometry; Citation Impact; PowerLaw; Self-Similar

JEL: Z210

Mathematics Code Classifications: 00A99

\section{INTRODUCCIÓN}

L a región de Latinoamérica y el Caribe, con un área aproximada de 8 millones de kilómetros cuadrados, representa $14 \%$ de la superficie terrestre. Su población de 560 millones de habitantes equivale a $8 \%$ de los habitantes del mundo. Asimismo, su economía aporta, aproximadamente, $8 \%$ del Producto Interno Bruto (PIB) global.

En los últimos 40 años, la producción científica total del sistema de ciencia de Latinoamérica y el Caribe ha crecido sostenidamente de manera exponencial (Figura 1). La producción científica latinoamericana y del Caribe se duplicaba cada 11 años; sin embargo, en este último periodo el crecimiento se triplicó en relación con la etapa previa. Entre las principales causas que han incidido en esta tendencia se encuentra la utilización del número de publicaciones como criterio principal para: 1) la asignación de fondos y recursos 
para la investigación, 2) la elección de académicos para integrar claustros de maestrías y doctorados, 3) la acreditación de programas de pre- y posgrado, 4) la contratación y promoción de académicos, 5) la ubicación de las universidades en rankings internacionales. Estos elementos han sido caracterizados en la metáfora del creador del ISI Web of Science, Eugene Garfield: "Las publicaciones y las citas que estas reciben son la divisa de los académicos" (Thomson Reuters, 2014).

\section{Producción científica total}

120.000

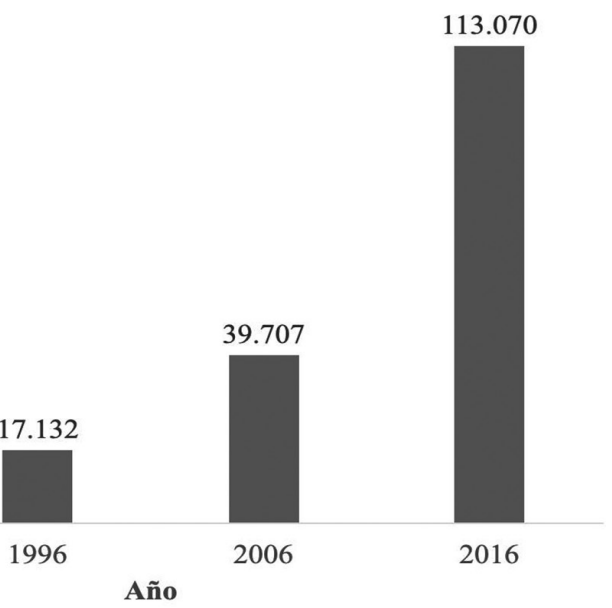

Año

Nota: los índices de citas considerados son Science Citation Expanded (SCI-Expandex), Social Science Citation Index (SSCI) y Arts \& Humanities Citation Index (A\&HCI). Incluye los documentos clasificados como "articles", "reviews", "letters" y "notes"

Figura 1. Tendencia en la producción científica latinoamericana y del Caribe. Se mide la producción científica total en el año

La importancia que se ha conferido a la producción y a la divulgación de nuevos conocimientos - a través de la publicación de artículos-implica que las instituciones que asignan recursos para la investigación, las que planifican y/o evalúan la política científica y las que la realizan cuenten con indicadores adecuados para evaluar el desempeño de los sistemas de ciencia a todos los niveles. Así, los usuarios que más requieren de dichos indicadores son los gobiernos que la financian y los ejecutores que necesitan obtener recursos para realizarla.

En la actualidad, la evaluación del desempeño científico desde el nivel individual (investigadores) hasta el global (países, regiones) se realiza mediante indicadores bibliométricos, construidos sobre la base del número de artículos publicados como indicador de producción y el número de citas 
recibidas como indicador de su impacto en la comunidad científica. Estos guarismos son, generalmente, aceptados por las comunidades científicas individuales. Para construir los indicadores bibliométricos se utilizan estadísticas basadas en la media y la desviación estándar (citas por artículo) e indicadores de rango de frecuencia como los índices de Hirsch (Hirsch, 2005) o de G (Egghe, 2013).

El empleo de indicadores basados en promedios, como el recuento del número de artículos y citas, presenta, entre otras, dos limitaciones importantes: 1) la dependencia del impacto del tamaño del área o disciplina en estudio (Van Raan, 2008; 2014), y 2) la asimetría de las distribuciones provenientes del recuento de las citas y los artículos (Newman, 2005; Clauset, Shalizi, y Newman, 2009; Katz, 2016). La primera se evidencia cuando en un área de investigación se publica un mayor número de artículos, por lo que poseerá un mayor impacto. La segunda limitante muestra la concentración de la producción en un pequeño grupo de áreas. Mientras que en un gran número de áreas de investigación se tendrá una producción científica escasa, un pequeño número de éstas acumularán $80 \%$ de la producción científica total. Similar tendencia se encontrará si se analiza el número de citas de estos campos.

La última limitación, la asimetría de la distribución, trae como consecuencia la formación de élites científicas que concentran la producción de conocimientos, y se caracteriza por distribuciones de leyes potenciales. En investigaciones se ha demostrado que el número de artículos publicados en revistas posee una distribución de ley de potencia, más conocida como Ley de Bradford (Naranan, 1989; Brookes, 1969; Bradford, 1946). De igual forma se ha establecido que las citas a los documentos siguen, aproximadamente, una ley de potencia (Clauset, Shalizi y Newman, 2009; Newman, 2005; Katz, 2005).

Por lo general, el exponente de las distribuciones de ley de potencia se ubica en un rango entre $2<\alpha<3$. La implicación práctica de este comportamiento es que, técnicamente, es incorrecto aplicar las estadísticas basadas en la media y en la desviación estándar, como el análisis de regresión lineal, ya que las poblaciones descritas por distribuciones con esta característica no poseen una media bien definida. Para superar esta limitación se deben emplear indicadores que cumplan con el requisito de invariancia de escala, con ello se evitan los sesgos en la evaluación de áreas o dominios de investigación. Los indicadores libres de escala poseen la ventaja de comparar sistemas de ciencia de vasta diferencia en tamaño sin el sesgo que introduce el volumen de los sistemas en comparación.

El objetivo de esta investigación es explorar el ritmo de crecimiento de la producción científica del sistema de ciencia latinoamericano y del Caribe en revistas de la región y su impacto. La implicación práctica de los resultados 
es mostrar la calidad de los artículos latinoamericanos y del Caribe en revistas dentro de la región. La pregunta a responder es la siguiente:

¿La producción científica de Latinoamérica y el Caribe en revistas de la región y su impacto crecen a un mismo ritmo?

Los resultados están dirigidos a 1) instituciones que financian la investigación en Latinoamérica y el Caribe, 2) los que formulan y evalúan la política científica, 3) grupos o cuerpos de evaluación de la ciencia y académicos de bibliometría, cienciometría, innovación y políticas públicas, y 4) los gestores de la ciencia en universidades y centros de investigación.

\section{ANTECEDENTES TEÓRICOS E HISTÓRICOS}

\section{Alometría y modelos alométricos}

La palabra alométrica proviene del griego allos, que significa "otro", otra métrica. En el lenguaje matemático se entiende como no lineal. Es decir, la relación no lineal entre dos variables analizadas. Se utiliza para encontrar una cantidad (exponente) que refleja la propiedad de invariancia de escala en una relación no lineal entre dos variables.

El modelo alométrico fue presentado por Julian Huxley en 1923, en su libro Problems of Relative Growth (Huxley, 1923). Posteriormente, en 1932 Max Kleiber lo utilizó en su estudio de la relación entre el metabolismo basal y el tamaño de los mamíferos, con lo que dio lugar a la Ley de Kleiber (Kleiber, 1932). En el campo de los análisis métricos de la ciencia, la cienciometría, este modelo tiene sus raíces en los estudios de Alfred Lotka, en 1925. Lotka investigó la distribución de la producción científica de los académicos del área de Física hasta 1900. Afirmó que seguía una ley de potencia con un exponente $\alpha \approx-2.02$. Estos resultados son conocidos como la Ley de Lotka (Lotka, 1926).

Posteriormente, De Solla-Price (1965) utilizó el modelo alométrico o de ley de potencia para analizar las redes de citas a los documentos científicos. Hoy se le denomina Ley de Price en honor al autor. En la década de 1970, el profesor Sundaresan Naranan desarrolló modelos matemáticos para analizar la presencia de la Ley de Bradford (Brookes, 1969; Bradford, 1946) en la distribución del número de artículos en las revistas científicas publicadas hasta 1970 (Naranan, 1970). El estudio corroboró que se comporta de acuerdo con una ley de potencia con un exponente $\alpha \approx-2$.0. En la década de 2000, el 
profesor Leo Egghe (Egghe, 2005) introdujo la llamada teoría lotkaiana para el estudio del proceso de producción de conocimientos, basada en el modelo alométrico o potencial.

El elemento más importante de la modelación alométrica es determinar el exponente que caracteriza la relación no lineal entre dos variables, considerada el valor cuantitativo de dicha relación. Este valor constituye la magnitud de invariancia de escala del sistema analizado o la presencia del Efecto Mateo (Merton, 1968). Dos variables que crecen exponencialmente en el tiempo en la forma $P=m e^{a t}$ e $I=n e^{b t}$ exhiben una relación alométrica entre $P$ e $I$ con un exponente dado por la razón de los exponentes de las variables en la forma $I \approx P^{n}$, donde $n=\frac{b}{a}$ (Sahal, 1981; Katz, 2005; Ronda-Pupo, 2017).

La principal ventaja del modelo alométrico es la propiedad de invariancia de escala, matemáticamente definida como $\frac{p(x)}{p(b x)}=g(b)$ para cualquier valor de $b$ (Newman, 2005). Esto es, si se incrementa la escala o las unidades de la medida de $x$ por un factor $b$, la forma de la distribución $p(x)$ no cambia, excepto si la misma se multiplica por una constante. La función de potencia en la forma general $p(x)=k x^{\alpha}$ en una correlación o $p(x)=k x^{-\alpha}$ en una distribución es la única función que posee la característica de invariancia de escala. Para el profesor Sundaresan Naranan (comunicación personal, 20 de octubre de 2018): "Las distribuciones de Ley de Potencia no son las únicas con la característica de invariancia de escala, aunque son las más simples. Otra posible distribución es la función de Weierstrass (Wikipedia, 2018) basada en series trigonométricas. Naranan y Balasubrahmanyan (2005) la describen como $\mathrm{F}(\mathrm{x})=\cos [2 \pi \mathrm{n}(\ln \mathrm{x} / \mathrm{lnb}]$. La cual muestra invariancia de escala $\mathrm{F}(\mathrm{x})=\mathrm{F}(\mathrm{bx})$. El índice $\mathrm{n}$ puede variar desde $-\infty$ hasta $+\infty)$ ".

En los últimos años se aprecia un incremento del uso de la modelación alométrica para analizar los sistemas de ciencia e innovación tecnológica, aunque su empleo en las ciencias sociales es menor (Coccia y Bozeman, 2016). Así, ha sido utilizada para estudiar las relaciones a escala entre el tamaño de las ciudades y su capacidad de innovación (Bettencourt et al., 2007), el impacto con el tamaño de los sistemas de ciencia (Katz, 2016), el impacto científico y la colaboración internacional (Coccia y Bozeman, 2016), o el tamaño de un sistema de innovación medido a través del volumen de producción científica y el impacto de dicho sistema (Ronda-Pupo, 2017).

Como se aprecia, el empleo del modelo o ecuación alométrica o de ley de potencia es un método en franco desarrollo para el estudio de los sistemas de ciencia e innovación, por no ser sensible a las variaciones del tamaño del sistema en análisis. Además, al ser capaz de establecer la invariancia de escala, el modelo alométrico es efectivo para analizar relaciones que provienen de distribuciones asimétricas donde el uso de medidas derivadas de promedios 
es, técnicamente, incorrecto. Asimismo, permite la generación de indicadores efectivos para comparar el desempeño de sistemas, disciplinas o áreas científicas de marcadas diferencias de tamaño.

El estudio aportará las características de invariancia de escala del crecimiento de la producción científica del sistema de ciencia de Latinoamérica y, específicamente, si el ritmo de aumento del impacto está acorde con el incremento de la productividad científica en revistas de la región.

\section{El estudio de las revistas latinoamericanas y del Caribe en el JCR}

Eugene Garfield, con su estudio "Journal Citation Studies 26. Latin American Journals" (Garfield, 1976), fue pionero al analizar el desempeño de las revistas latinoamericanas y del Caribe. Este trabajo es el más citado entre las investigaciones sobre ellas (115 citas $=17 \%$ del total). Una búsqueda rápida en WoS devuelve que entre enero de 1976 y noviembre de 2018 se publicaron 175 artículos sobre el tema. De ellos, 17 aparecen en la categoría Information Science \& Library Science de WoS. Scientometrics acumula la mayor cantidad de documentos sobre este tema (seis).

La Figura 2 muestra el crecimiento del interés académico sobre las revistas latinoamericanas y del Caribe. Resulta significativo que $75 \%$ de los estudios sobre esta temática se han publicado en los últimos 15 años. Se confirma el crecimiento sostenido del número de revistas indexadas en el JCR (Collazo-Reyes, 2014), porque ha influido, significativamente, en los patrones de publicaciones y citaciones de los países de la región (Collazo-Reyes et al., 2008; Gómez et al., 1999).

En los últimos años se ha incrementado el interés en el estudio de las revistas latinoamericanas y del Caribe indexadas en las principales bases de datos internacionales. Así, se ha analizado la percepción de editores y autores sobre la estrategia editorial de acceso abierto de las revistas de la región (Sánchez-Tarragó et al., 2016), al igual que el análisis bibliométrico de la producción de revistas científicas de América Latina y del Caribe en Scopus, Journal Citations Reports y Latindex, en el campo de los recursos naturales. El incremento del interés por mejorar la calidad de las revistas regionales puede estar asociado con la necesidad imperante de aumentar la visibilidad de las instituciones como vía favorecedora para escalar en el posicionamiento en los rankings internacionales. Unos de los métodos para elaborar dicha clasificación es el criterio bibliométrico, específicamente los números de publicaciones en revistas de circulación internacional y de citas a los trabajos de los académicos. 


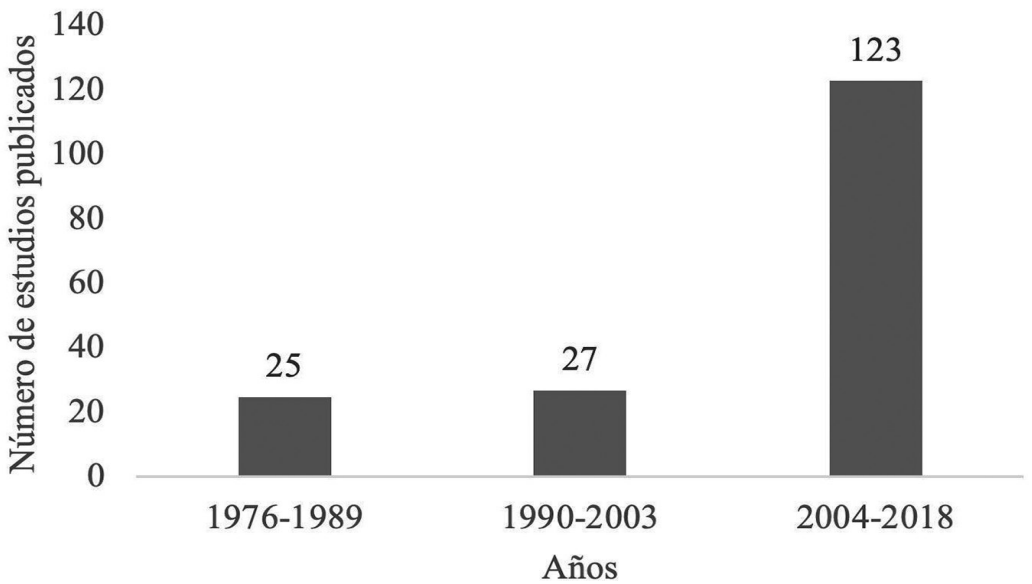

Figura 2. Número de estudios publicados en WoS relacionados con las revistas latinoamericanas y del Caribe Fuente: Journal of Citation Reports, edición 2017

\section{La distribución por países de las revistas latinoamericanas y del Caribe en el JCR}

La Tabla 1 presenta la distribución del número de revistas latinoamericanas y del Caribe por países. El resultado sugiere la presencia del Efecto Mateo. Sólo siete países (15\%) de los 46 de Latinoamérica y el Caribe hospedan revistas que aparecen en el JCR. Brasil acumula $50.4 \%$ del total de ellas, seguido por México (16\%) y Chile (15.2\%). Luego parecen Argentina (7.8\%), Colombia (7 \%) y Venezuela (3.3 \%). Coincide que estos países mostraron un mayor desarrollo económico en los pasados 40 años. Este comportamiento sugiere que la generación de conocimientos científicos pudiera estar ligada a la tenencia de recursos destinados a incrementar la investigación e innovación.

Un aspecto a considerar es que el número de revistas latinoamericanas y del Caribe indexadas en WoS se incrementó de 69 a 248 entre 2006 y 2009 (Collazo-Reyes, 2014). Sin embargo, sólo 18 \% de la producción científica de Latinoamérica y el Caribe aparece en dichas revistas.

\begin{tabular}{|l|c|c|c|c|c|c|}
\hline \multicolumn{1}{|c|}{ País } & SCIE & \% de 195 & SSCI & \% de 69 & $\begin{array}{c}\text { Total JCR sin } \\
\text { duplicados }\end{array}$ & \% de 244 \\
\hline Brasil & 112 & $57.4 \%$ & 23 & $33.3 \%$ & 123 & $50.4 \%$ \\
\hline México & 25 & $12.8 \%$ & 14 & $20.3 \%$ & 39 & $16 \%$ \\
\hline Chile & 23 & $11.8 \%$ & 18 & $26.1 \%$ & 37 & $15.2 \%$ \\
\hline
\end{tabular}




\begin{tabular}{|l|c|c|c|c|c|c|}
\hline Argentina & 15 & $7.7 \%$ & 5 & $7.2 \%$ & 19 & $7.8 \%$ \\
\hline Colombia & 12 & $6.2 \%$ & 7 & $10.1 \%$ & 17 & $7 \%$ \\
\hline Venezuela & 7 & $3.6 \%$ & 2 & $2.9 \%$ & 8 & $3.3 \%$ \\
\hline Costa Rica & 1 & $0.5 \%$ & 0 & $0 \%$ & 1 & $0.4 \%$ \\
\hline $\begin{array}{c}\text { Total de la } \\
\text { región LAC }\end{array}$ & 195 & $100 \%$ & 69 & $100 \%$ & 244 & $100 \%$ \\
\hline
\end{tabular}

Nota: SCIE = Science Citation Index Empanded. SSCI = Social Science Citation Index. En el total no se contemplan las revistas que pertenecen a ambos índices de citas

Tabla 1. Número de revistas de Latinoamérica y el Caribe indexadas en el JCR por países Fuente: Journal of Citation Reports, edición 2017

\section{Metodología}

\section{El modelo}

Para el estudio se utilizó la ecuación alométrica o de ley de potencia:

$$
Y=\gamma X^{\alpha}
$$

donde $Y$ representa el impacto, $X$ la producción científica. $\gamma$ y $\alpha$ son constantes. $\gamma$ es la constante de normalización y $\alpha$ es el exponente o pendiente de la línea de regresión de las variables en escala logarítmica. La transformación logarítmica de la ecuación 2 resulta en una línea (Huxley, 1923).

$$
\log (Y)=\alpha \log (X)+\log (\gamma)
$$

Se utilizó el método de Mínimos Cuadrados Ordinarios (MCO) para el ajuste de la línea de regresión. Se seleccionó este método por ser el que realiza el ajuste con el mínimo de error (Legendre y Legendre, 2012). Asimismo, el procedimiento MCO es el adecuado cuando el objetivo del estudio es la predicción de la variable dependiente a partir de la variable independiente (Smith, 2009).

Los requisitos para la prueba estadística son los siguientes: 1) la normalidad de la distribución de los datos alrededor de la línea de regresión, 2) la varianza de la variable dependiente en la población es constante, aparte del valor de la variable independiente, y 3 ) independencia de los residuos.

El exponente $\alpha$ indica el patrón de crecimiento, invariancia de escala o Efecto Mateo. Su valor se puede ubicar en uno de tres posibles rangos.

$$
\alpha=1
$$


Cuando el impacto y la producción científica crecen a un mismo ritmo, no existe ventaja acumulativa de una variable con respecto a la otra. Para los investigadores de las comunidades de Biología y Ecología, se dice hay una relación isométrica entre las variables analizadas (Warton et al., 2006).

$$
\alpha<1
$$

Cuando $\alpha<1$, la variable que ocupa la posición de $X$ (producción) crece a un ritmo más lento que la variable $Y$ (impacto). Existe una relación sublineal o alométrica negativa.

$$
\alpha>1
$$

Finalmente, cuando $\alpha>1$, la variable que ocupa la posición de $X$ (producción) crece a un ritmo más rápido que la variable que ocupa la posición de $Y$ (impacto). Esto es, hay una relación súper lineal y una ventaja acumulativa positiva de la variable $X$ con respecto a la variable $Y$. También se interpreta como que existe una relación alométrica positiva.

\section{Los datos}

Los documentos de las categorías artículo, revisión, notas y cartas publicados en revistas donde, al menos, un autor pertenece a una institución ubicada en un país latinoamericano o del Caribe constituyen las fuentes de información para el estudio. Una condición indispensable es que cada revista-fuente debe aparecer en el Journal of Citation Reports, edición 2017, y ubicarse en un país latinoamericano o del Caribe. Para obtener la información se realizó una búsqueda avanzada en la base de datos Web of Science, en los indices de citas Science Citation Expanded (SCI-Expandex), Social Science Citation Index (SSCI) y Arts \& Humanities Citation Index (A \& HCI) en el rango temporal de 2000 hasta 2017, inclusive.

\section{Las variables del modelo}

\section{Producción científica (P)}

Para el estudio se definió producción científica como el número de documentos publicados por autores de instituciones de países de Latinoamérica y el Caribe en revistas de la región en las bases de datos de citaciones Science Citation Expanded (SCI-Expandex), Social Science Citation Index (SSCI) y 
Arts \& Humanities Citation Index (A \& HCI). Por “de la región” se entiende que esté ubicada en uno de los países de la región latinoamericana y del Caribe. Para la recolección de los datos se utilizaron los documentos de las categorías 'article', 'review', 'letter' y 'notes'.

\section{El impacto (I)}

Se define impacto como el número de citas que recibieron los documentos latinoamericanos y del Caribe publicados en revistas de la región durante el marco temporal definido. En nuestro caso se utilizó una ventana temporal de tres años. Esto es, el conteo de las citas a cada documento en el año de publicación, más la suma de ellas recibidas en los dos años siguientes. Por ejemplo, el impacto de un artículo publicado en 2017 será la suma de las citas recibidas en el año, más las citas en 2018 y 2019; así sucesivamente.

Para el cálculo del impacto de cada documento se creó un informe de citas a los artículos publicados en cada año del marco temporal estudiado. La base de datos WoS sólo realiza el informe de citas de 10000 registros máximo. Entre 2008 y 2017 inclusive la cantidad de documentos publicados excede esa cifra. Por ello, fue necesario agregar los registros a la lista de registros marcados en grupos de 10 000, realizar el informe de citas de esta cantidad, y repetir el proceso hasta concluir el cálculo del impacto de la totalidad de artículos publicados en cada año. Por restricciones de WoS, los resultados se descargaron en grupos de 500, en formato Excel, y se almacenaron en una base de datos en Structured Query Language (SQL). Finalmente, se calculó el impacto de cada documento para cada año. La utilización de una ventana fija de citaciones elimina el sesgo que produce la fluctuación de citas ocasionada por el tiempo de la publicación y el factor de impacto de las revistas.

\section{Presentación y ANÁlisis De los Resultados}

\section{La producción científica de Latinoamérica y el Caribe en revistas JCR de la región}

La Tabla 2 muestra la producción científica latinoamericana y del Caribe y el impacto entre 2000 y 2017. El resultado evidencia un patrón de crecimiento a través de los años hasta 2010. En adelante, la productividad se ha estabilizado en torno a los 14000 artículos anuales. Este resultado sugiere la posible existencia de una correlación entre la cantidad de revistas y el número de artículos publicados. Este resultado debe ser corroborado para determinar en 
cuánto se incrementaría la producción científica con el aumento del número de revistas en dicha área.

\begin{tabular}{|l|r|r|}
\hline Año de publicación & Producción & Impacto $(\mathbf{t} 0+3)$ \\
\hline 2000 & 3.222 & 2.135 \\
\hline 2001 & 2.977 & 2.241 \\
\hline 2002 & 3.184 & 2.682 \\
\hline 2003 & 3.786 & 3.268 \\
\hline 2004 & 4.009 & 3.817 \\
\hline 2005 & 4.361 & 4.958 \\
\hline 2006 & 4.983 & 6.192 \\
\hline 2007 & 9.208 & 11.074 \\
\hline 2008 & 12.690 & 15.218 \\
\hline 2009 & 13.842 & 18.002 \\
\hline 2010 & 14.404 & 18.434 \\
\hline 2011 & 15.383 & 19.715 \\
\hline 2012 & 14.976 & 18.819 \\
\hline 2013 & 14.842 & 19.345 \\
\hline 2014 & 14.531 & 19.868 \\
\hline 2015 & 14.360 & 20.772 \\
\hline 2016 & 14.132 & 22.772 \\
\hline 2017 & 14.329 & 24.040 \\
\hline Total & 179.219 & 233.352 \\
\hline
\end{tabular}

Nota: se utilizó el número de revistas latinoamericanas y del Caribe que aparecen en el reporte JCR, edición 2017

Tabla 2. Producción científica e impacto de Latinoamérica y el Caribe en revistas de la región Fuente: artículos publicados en revistas de Latinoamérica y el Caribe en los índices de citas Science Citation Index Expanded, Social Science Citation y Art \& Humanities Citation Index

\section{La relación alométrica entre el impacto y la producción científica}

Los supuestos que se deben cumplir para la regresión alométrica: normalidad Shapiro Wilk $=0,95 ; p=0,46$, varianza constante $p=0,66$. Se utilizó un valor $p<0,01$ para rechazar las hipótesis de la distribución y la varianza constante. Asimismo, los valores de criterio de selección del modelo utilizado $R^{2}>0,90$ 
y el estadístico predictor de error PRESS =0,03 confirman que existe un buen ajuste de la línea de regresión a la ecuación alométrica utilizada. La prueba estadística Predicted Residual Error Sum of Squares (PRESS) mide cuán bien la ecuación utilizada se ajusta a los datos reales de la regresión.

La Figura 3 muestra los resultados de la correlación alométrica realizada. El exponente $\alpha=0,75 \pm 0,02$ resultante, utilizando producción científica como variable ' $X$ ', sugiere que el impacto crece a un ritmo inferior que la producción científica. En términos prácticos, esto sugiere que la producción científica latinoamericana y del Caribe en las revistas de la región crece a un ritmo más rápido que el número de citas (impacto) que reciben estas publicaciones. Se incrementa el número de documentos, pero la cantidad de citas que reciben aumenta en una proporción menor.

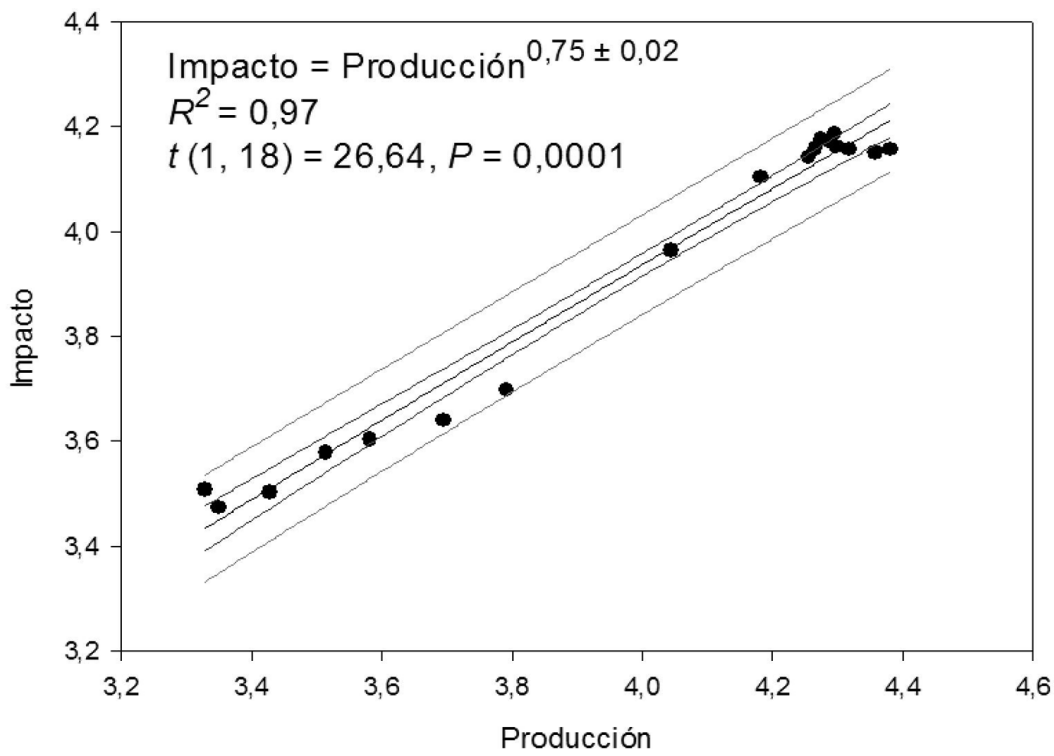

Figura 3. Resultados de la correlación alométrica en escala logarítmica, considerando el impacto como variable ' $Y$ '

La estadística $t$ de Student se utilizó para confirmar la significación de los resultados del modelo alométrico, también para analizar el ritmo de crecimiento de la producción científica y su impacto. La estadística $t$ de Student fue $t(1,18)=26,64, p=0,0001$. El valor difiere estadísticamente de 0 , lo que confirma la significación de la relación no lineal entre ambas variables. Para comprobar la existencia de una verdadera correlación alométrica o de ley de potencia, se siguió a Legendre y Legendre (2012: 550, ecuación 10.13). El procedimiento sugerido por estos autores utiliza los parámetros $\alpha$ (exponente de 
la relación) y $r$ (correlación de Pearson) de Mínimos Cuadrados Ordinarios para calcular la correlación de escala a través del Eje Mayor Estandarizado (SMA), por sus siglas en inglés. $\operatorname{Si} \alpha_{\mathrm{SMA}} \approx \alpha_{\mathrm{OLS}}$ se concluye que las variables están altamente correlacionadas (ecuación 3). Cuando no existe una correlación, siempre $\alpha_{\mathrm{SMA}}>\alpha_{\mathrm{OLS}}$. El resultado para la correlación analizada muestra que $\alpha_{\mathrm{SMA}}=\alpha_{\mathrm{OLS}}$. El resultado respalda la existencia de una correlación acorde a una ley de potencia o alométrica entre la producción científica del sistema de ciencia latinoamericano y del Caribe en revistas de la región y su impacto.

$$
\alpha_{S M A}=\frac{\left|\alpha_{O L S}\right|}{r_{x y}}, \text { when } r_{x y} \neq 0
$$

\section{DisCUSIÓN Y CONSIDERACIONES FINALES}

El resultado obtenido muestra que el impacto basado en citaciones del sistema de ciencia latinoamericano y del Caribe en revistas dentro de la región crece a un ritmo más lento que la productividad $\alpha<1$. Para comprobar que el exponente menor que la unidad no obedece al uso de una ventana temporal muy pequeña, $t_{0+2}$, se corrió la prueba para un rango temporal de ventanas fijas de citaciones desde tres años hasta los ocho. El exponente del impacto se mantuvo en torno a 0,60 $\pm 0,05$ durante todo el rango temporal, lo que asegura que el resultado no obedece a un patrón de lentitud en las citaciones.

El incremento del número de revistas ha impulsado el aumento de la producción científica latinoamericana y del Caribe (Collazo-Reyes, 2014). Sin embargo, un gran número de los artículos publicados no recibe citas (47\%) o logra una (24\%). Sólo $16 \%$ posee dos o más citas. Este comportamiento confirma la existencia de una desventaja acumulativa del impacto de las publicaciones en revistas latinoamericanas y del Caribe con respecto de su productividad. La evidencia empírica del estudio sugiere que las revistas latinoamericanas y del Caribe están condenadas a mantenerse en el último cuartil del JCR. En un estudio en curso se compararán los ritmos de crecimiento de la producción y el impacto del sistema de ciencia de Latinoamérica en revistas de la región en relación con su producción en publicaciones foráneas.

Entre las principales causas de la desventaja acumulativa del impacto del sistema de ciencia latinoamericano y del Caribe en revistas de la región se pueden enunciar: la percepción de los autores con respecto a la calidad de las revistas de la región; el idioma en que aparecen publicados los artículos, y el ámbito de interés del artículo, regional o externo.

En cuanto a la percepción de los autores con respecto a la calidad de las revistas, esto es, por el cuartil de ubicación, sólo 2 \% (cuatro) de las publicaciones 
incorporadas en JCR se ubica en el primer cuartil y $6 \%$ (14) en el segundo. Este elemento se convierte en una restricción pues las instituciones exigen a sus académicos colocar su producción científica en revistas con el mayor factor de impacto, ubicadas, principalmente, en Norteamérica o Europa. Esto se confirma porque 89 \% de los artículos de autores latinoamericanos y del Caribe aparecen en revistas foráneas. En este sentido, estudios sugieren políticas para incrementar la calidad de las revistas latinoamericanas y del Caribe, como el acceso abierto (Estrada-Mejía y Forero-Pineda, 2010; Sánchez-Tarragó et al., 2016). Asimismo, la disminución de tiempos en revisión de pares y el fortalecimiento de los comités editoriales pudiera contribuir al mejoramiento de la calidad de las revistas de la región.

El idioma es otro factor a tener presente. El $71 \%$ de las revistas latinoamericanas y del Caribe incorporadas en el JCR publica sus artículos en español o portugués y $29 \%$ en inglés. Se conoce por estudios que los artículos en inglés tienden a ser leídos por una comunidad más amplia y, por ello, la probabilidad de atraer citas es mayor (Cremades Pallas et al., 2013; Dewey, 2007). Los autores tienden a colocar sus mejores resultados en revistas con un público objetivo más amplio. Generalmente, esas publicaciones circulan en inglés. El 81 \% de la producción científica total del sistema de ciencia de Latinoamérica se publica en esta lengua.

El interés regional versus el interés externo también pudiera tener relación en el lento crecimiento del impacto de la productividad latinoamericana y el Caribe en revistas dentro de la región. Collazo-Reyes et al. (2008) reportaron la existencia de un bajo flujo de citas entre documentos de interés más regional, mientras que los aportes de autores de fuera de la región, publicados en inglés, reciben mayor atención y citaciones.

Los resultados se verían favorecidos si se ampliara la cantidad de antecedentes al incorporar otras bases de datos, como Scopus y Scielo. Nuestra investigación abre nuevas interrogantes, tales como: ¿existe una relación súper lineal de la producción científica de un área y el número de revistas en la misma? ¿En cuánto se incrementaría la producción científica de un área de investigación si se duplica el número de publicaciones? ¿El impacto de la producción científica latinoamericana y del Caribe en revistas fuera de la región es superior o inferior al impacto de las ubicadas dentro de la misma? 
El autor agradece a tres evaluadores de la revista Investigación Bibliotecológica: archivología, bibliotecología e información, cuyos comentarios contribuyeron a mejorar los resultados de la investigación. El presente resultado se dedica a la memoria del profesor Sundaresan Naranan quien falleció en la

India en julio de 2019.

Información sobre financiamiento

Investigación financiada a través del proyecto $\mathrm{N}^{\circ} 1180200$ del Fondo Nacional de Desarrollo Científico y Tecnológico (FONDECYT), Chile, 2018.

\section{REFERENCIAS}

Bettencourt, L. M. A., J. M. Lobo, D. Helbing, C. Kuhnert y G. B. West. 2007. "Growth, innovation, scaling, and the pace of life in cities". Proceedings of the National Academy of Sciences of the United States of America 104 (17): 7301-7306. https://doi.org/10.1073/pnas.0610172104

Bradford, S. C. 1946. Documentation. London: Crosby Lockwood.

Brookes, B. C. 1969. "Bradford's law and the bibliography of science". Nature 224 (5223): 953-956. https://doi.org/10.1038/224953a0

Clauset, A., C. R Shalizi y M. E. J. Newman. 2009. "Power-law distributions in empirical data”. SIAM Review 51 (4): 661-703. https://doi.org/10.1137/070710111

Coccia, M. y B. Bozeman. 2016. "Allometric models to measure and analyze the evolution of international research collaboration”. Scientometrics 108 (3): 1065-1084. https://doi.org/ 10.1007/s11192-016-2027-x

Collazo-Reyes, F. 2014. "Growth of the number of indexed journals of Latin America and the Caribbean: the effect on the impact of each country". Scientometrics 98 (1): 197-209. https://doi.org/10.1007/s11192-013-1036-2

Collazo-Reyes, F., M. E. Luna-Morales, J. M. Russell y M. A. Pérez-Angón. 2008. "Publication and citation patterns of Latin American \& Caribbean journals in the SCI and SSCI from 1995 to 2004”. Scientometrics 75 (1): 145-161. https://doi.org/10.1007/s11192-007-1841-6

Cremades Pallas, R., P. Burbano, M. A. Valcárcel de La Iglesia, G. Burillo-Putze, F. J. Martín-Sánchez y Ò. Miró. 2013. "Impacto de la inclusión de artículos escritos en inglés en revistas biomédicas españolas de edición multilingüe”. Anales del Sistema Sanitario de Navarra 36 (3): 467-470. https://doi.org/10.4321/s1137-66272013000300011

De Solla-Price, D. J. 1965. "Networks of Scientific Papers". Science 14 (3683): 510-515. https://doi.org/10.1126/science.149.3683.510

Dewey, M. 2007. "English as a lingua franca and globalization:an interconnected perspective". International Journal of Applied Linguistics 17 (3): 332-354. https://doi.org/10.1111/j.1473-4192.2007.00177.x 
Egghe, L. 2005. Power Laws in the Information Production Process: Lotkaian Informetrics. UK: Elsevier Academic Press.

Egghe, L. 2013. "Theory and practise of the g-index". Scientometrics 69 (1): 131-152. https://doi.org/10.1007/s11192-006-0144-7

Estrada-Mejía, C., y C. Forero-Pineda. 2010. "The quest for visibility of scientific journals in Latin America”. Learned Publishing 23 (3): 237-252. https://doi.org/10.1087/20100306

Garfield, E. 1976. "Journal Citation Studies. 26. Latin-American Journals”. Current Contents (37): 5-11.

Gómez, I., R. Sancho, L. Moreno y M. T. Fernandez. 1999. "Influence of Latin American journals coverage by international databases”. Scientometrics 46 (3): 443-456. https://doi.org/ 10.1007/bf02459603

Hirsch, J. E. 2005. "An index to quantify an individual's scientific research output". Proceedings of the National Academy of Sciences of the United States of America 102 (46): 16569-16572. https://doi.org/10.1073/pnas.0507655102

Huxley, J. S. 1923. Problems of Relative Growth. London: Methuen \& Co. LTD.

Katz, J. S. 2005. "Scale-independent bibliometric indicators". Measurement 3 (1): 24-28. https://doi.org/ 10.1207/s15366359mea0301_3

Katz, S. 2016. “What is a Complex Innovation System?” Plos One 11 (6): e0156150. https://doi.org/10.1371/journal.pone.0156150

Kleiber, M. 1932. “Body size and metabolism”. Hilgardia 6:315-353.

Legendre, P. y L. Legendre. 2012. Numerical Ecology. 3 ed. Vol. 24. Great Britain: Elsevier B. V. Developments in Environmental Modeling.

Lotka, A. J. 1926. "The frecuency distribution of scientific productivity". Journal of the Washington Academy of Sciences 16 (12): 317-323. http://www.jstor.org/stable/i24527553

Merton, R. K. 1968. "The Matthew Effect in Science”. Science 159 (3810): 56-63. https://doi.org/10.1126/science.159.3810.56

Naranan, S. 1970. "Bradford's law of bibliography of science: an interpretation". Nature 227 (5258): 631-632. https://doi.org/10.1038/227631a0

Naranan, S. 1989. "Power Law Version of Bradford Law - Statistical Tests and Methods of Estimation”. Scientometrics 17 (3-4): 211-226. https://doi.org/10.1007/Bf02026411

Naranan, S. y V. K. Balasubrahmanyan. 2005. Power Laws in Statistical Linguistics and related Systems. An International Handbook of Quantitative Linguistics. Berlin: Walter de Gruyter.

Newman, M. E. J. 2005. "Power laws, Pareto distributions and Zipf's law". Contemporary Physics 46 (5): 323-351. https://doi.org/10.1080/00107510500052444

Ronda-Pupo, G. A. 2017. "The citation-based impact of complex innovation systems scales with the size of the system". Scientometrics 112 (1): 141-151. https://doi.org/10.1007/s11192-017-2401-3

Ronda-Pupo, G. A. y J .S. Katz. 2017. “The Scaling Relationship between Citation-Based Performance and Scientific Collaboration in Natural Sciences". Journal of the Association for Information Science and Technology 68 (5): 1257-1265. https://doi.org/10.1002/asi.23759 
Sahal, D. 1981. Patterns of technological innovation. New York: Addison-Wesley.

Sánchez-Tarragó, N., A. Caballero-Rivero, P. Trzesniak, D. Deroy Domínguez, R. N. M. dos Santos y J. C. Fernández-Molina. 2016. "Scientific journals in Latin America on their way towards open access: A diagnosis on policies and editorial strategies". Transinformação 28 (2): 159-172. https://doi.org/10.1590/2318-08892016000200003

Smith, R. J. 2009. "Use and misuse of the reduced major axis for line-fitting". American Journal of Physics and Anthropology 140 (3): 476-486. https://doi.org/10.1002/ajpa.21090

Thomson Reuters. 2014. 50 Years of Citation Indexing: A visit with Dr. Eugene Garfield. 13 de mayo. Video de Youtube, 5:48. https://www.youtube.com/watch?v=2kZ0_5HTYDQ

Van Raan, A. F. J. 2008. "Scaling rules in the science system: Influence of field-specific citation characteristics on the impact of research groups". Journal of the American Society for Information Science and Technology 59 (4): 565-576. https://doi.org/10.1002/asi.20765

Van Raan, A. F. J. 2014. "Bibliometrics: Use and abuse in the review of research performance", in Advances in bibliometric analysis: Research performance assessment and science mapping, editado por W. Blockmans, L. Engwall y D. Weaire, 17-28. London, UK: Portland Press.

Warton, D. I., I. J. Wright, D. S. Falster y M. Westoby. 2006. "Bivariate line-fitting methods for allometry”. Biological Reviews Cambridge Philosofical Society 81 (2): 259-291. https://doi.org/10.1017/S1464793106007007

Wikipedia. 2018, s. v. "Función de Weierstrass". Revisado el 11 de diciembre de 2018. https://es.wikipedia.org/wiki/Funci\%C3\%B3n_de_Weierstrass

Para citar este texto:

Ronda-Pupo, Guillermo Armando. 2021. "Producción científica e impacto del sistema de ciencia de Latinoamérica y el Caribe en revistas de la región". Investigación Bibliotecológica: archivonomía, bibliotecología e información 35 (88): 45-62.

http://dx.doi.org/10.22201/iibi.24488321xe.2021.88.58358 